\title{
Consumo de drogas em contextos rurais: revisão sistemática na América Latina e Caribe
}

\section{Drug Use in Rural Contexts: Systematic Review in Latin America and Caribbean}

\author{
Pedro Barino Rodrigues (orcid.org/0000-0002-6794-743X) ${ }^{1}$ \\ Telmo Mota Ronzani (orcid.org/0000-0002-8927-5793)2,*
}

\begin{abstract}
Resumo
Este estudo objetivou clarificar o cenário de consumo de drogas em contextos rurais na América Latina e Caribe. Trata-se de uma revisão sistemática seguindo o protocolo Prisma nas bases de dados Lilacs, PePSIC, PsycINFO/PsycNET, PubMed e SciELO. A amostra final foi composta por 20 artigos, os resultados indicam uma prevalência em enxergar o "problema das drogas" a partir de uma perspectiva epidemiológica, mas que existem críticas e outras construções com visões mais integrativas; os contextos rurais se mostraram heterogêneos e perpassados por questões socioeconômicas. Nesse sentido, discute-se um olhar em que a história, a cultura, a geografia, o território e a população são fundamentais para a percepção dos agentes nesses contextos.
\end{abstract}

Palavras-chave: Revisão sistemática. Drogas. Zona rural. Transtornos relacionados ao uso de substâncias. População rural.

\begin{abstract}
This study aimed to clarify the drug use in rural contexts in Latin America and Caribbean. This is a systematic review following the Prisma protocol in the databases: Lilacs, PePSIC, PsycINFO/PsycNET, PubMed e SciELO. The final sample consisted of 20 articles; the results indicate a prevalence in considering the "drug problem" from an epidemiological perspective; however, criticism and other constructions with more integrative visions still exist; the rural contexts are heterogeneous and crossed by socioeconomic issues. In this sense, we discussed a view in which history, culture, geography, territory and population are essential for the perception of agents in these contexts.
\end{abstract}

Keywords: Systematic review. Drugs. Rural areas. Substance-related disorders. Rural population.

\footnotetext{
${ }^{1}$ Universidade Federal de Juiz de Fora, Juiz de Fora, Brasil. E-mail: barino92@gmail.com.

2 Universidade Federal de Juiz de Fora, Juiz de Fora, Brasil. E-mail: tm.ronzani@gmail.com.

* Bolsista de Produtividade CNPq 1D.
} 
Segundo o Escritório das Nações Unidas sobre Drogas e Crime (UNODC, 2017), o consumo de drogas é tradicionalmente visto como urbano, um problema inerente à cidade. Porém, nos últimos anos, percebeu-se um aumento na prevalência de Transtornos Relacionados ao Uso de Substâncias e da mortalidade relacionada ao consumo em regiões rurais. No entanto, como apontado no mesmo estudo, as evidências na América Latina e principalmente na América do Sul ainda não são claras.

O III Levantamento Nacional sobre o Uso de Drogas pela População Brasileira (Fiocruz, 2017), por exemplo, aponta que em relação ao consumo de álcool e substâncias ilícitas não houve diferenças estatisticamente significativas na comparação entre contextos rurais e urbanos. Para além dos dados da prevalência de consumo, é importante considerar que o impacto das condições de saúde, de uma maneira geral, tem uma importante relação com algumas determinações sociais (Galvão, Finkelman \& Henao, 2011); entre elas destacam-se as barreiras de acesso, sejam elas culturais, sejam geográficas, e determinações de classe, mais especificamente o impacto do consumo de drogas em situações de pobreza (Mendes, Ronzani \& Paiva, 2019).

No Brasil, aproximadamente $16 \%$ da população vive em áreas rurais (IBGE, 2017), podendo haver um número maior se considerarmos alguns municípios de pequeno porte e com características suburbanas (Savassi, Almeida, Floss \& Lima, 2018). Além disso, sabe-se que há uma lacuna em relação ao acesso aos cuidados e um deficit nos indicadores de saúde de uma maneira geral para essa população. Soma-se a isso uma baixa cobertura do cuidado, a deficiência de políticas públicas específicas para essa população e uma formação em saúde eminentemente urbana (Ronzani, Mendes, Oliveira \& Leite, 2019).

Apesar de muitos trabalhos abordarem a questão das drogas como um problema em si, já se sabe que a percepção das drogas perpassa por processos históricos e culturais. Dessa forma, não se nega os problemas potenciais em relação ao biológico, psicológico e social, mas devemos nos atentar à necessidade e importância de uma visão mais integrativa, considerando fatores médio e macrossociais (Ronzani, 2018). No Brasil, por exemplo, as políticas públicas chegam ao campo com suas limitações, mas os profissionais não compreendem bem esses territórios, uma vez que não têm experiências em sua formação 
em contextos rurais, sendo comum ter o primeiro emprego a partir dessas políticas (Silva $\&$ Macedo, 2017). O conceito de território é amplo e estudado por diferentes campos do conhecimento e tem sido usado de forma mais consensual nas políticas públicas, ganhado relevância na saúde pública e tornando-se uma ferramenta útil para a compreensão, planejamento e operacionalização de intervenções reais na vida das pessoas (Dimenstein, Dalla Vecchia, Macedo \& Bastos, 2018).

As barreiras de acesso geográfico, com longas distâncias e acessos de baixa qualidade aos centros urbanos e serviços de saúde, são marcantes nos contextos rurais. Os serviços de tratamento e de acesso à saúde são localizados nas áreas urbanas e o acesso a esses serviços tem como obstáculo o alto custo das viagens e as poucas opções de transporte público (Savassi et al., 2018). Além disso, há ainda uma importante limitação para o acesso a algumas tecnologias que poderiam facilitar o cuidado em saúde, tais como a utilização de internet ou cuidados em saúde a distância (UNODC, 2017).

Assim, o consumo de substâncias entre essa população é agravado por alguns marcadores importantes, tais como os socioeconômicos, acesso, infraestrutura, falta de formação profissional para atuar na área e ausência de políticas públicas específicas, o que pode tornar mais grave as condições de saúde de pessoas com problemas devido ao uso de álcool e outras drogas (UNODC, 2017). Ainda, segundo o World Drug Report (UNODC, 2016), nos últimos anos, o número de pessoas que fazem uso abusivo de drogas no âmbito rural subiu desproporcionalmente pela primeira vez desde 2010 no mundo (mais de 29 milhões de pessoas). Portanto, torna-se fundamental o planejamento de políticas públicas e intervenções baseadas no contexto e o conhecimento e sistematização sobre os avanços e lacunas na área.

Sendo assim, o objetivo do presente estudo é realizar uma revisão sistemática da literatura sobre o consumo de drogas em contextos rurais na América Latina e Caribe. Espera-se contribuir com a área, a partir da apresentação dos principais avanços e lacunas sobre o tema.

\section{Metodologia}


Realizou-se uma revisão sistemática de literatura, baseada no protocolo Preferred Reporting Items For Systematic Reviews and Meta-Analyses - Prisma (Liberati et al., 2009). Foi utilizada a tradução em português mais recente e licenciada pelo grupo Prisma para facilitar e sistematizar ainda mais o processo. O objetivo do Prisma é ajudar os autores a melhorarem o relato de revisões sistemáticas e meta-análises a partir de um checklist com 27 itens e um fluxograma de quatro etapas (Galvão et al., 2015).

A busca ocorreu entre meados de junho e setembro de 2018. As bases consultadas foram Lilacs, PePSIC, PsycINFO/PsycNET, PubMed e SciELO. O critério de definição se deveu por serem as principais bases da literatura científica no campo da Saúde e Psicologia, com os maiores repositórios de artigos no mundo e em diferentes idiomas. Para se realizar a busca e definir os termos de forma mais precisa, foi utilizado o Medical Subject Headings (MeSH) utilizando dois termos chave: "rural" $\times$ "dependência de drogas". Assim, foram encontrados dois termos para o primeiro lado do cruzamento: "população rural" e "zona rural", com suas devidas traduções em inglês e espanhol - "rural population"/ "rural areas" e "población rural"/"medio rural" -; e do outro lado do cruzamento, seis termos: "transtornos relacionados ao uso de substâncias", "drogas", "alcoolismo", "álcool”, "tabagismo" e "tabaco", também com suas respectivas traduções - "substance-related disorders"/"transtornos relacionados con sustancias", "drugs"/drogas", "alcoholism"/"alcoholismo", "alcohol"/"alcohol", "tobacco use disorder"/"tabaquismo" e "tobacco"/"tabaco".

Foram utilizados alguns critérios de inclusão: a) o artigo deveria ser empírico, eliminando teses, revisões sistemáticas, monografias, trabalhos de conclusão de curso etc.; b) deveria tratar claramente do tema abordado pelos objetivos do trabalho, eliminando estudos que falavam, por exemplo, de doenças crônicas ou respiratórias que teriam drogas como fator associado ou secundário; c) escritos em português, inglês ou espanhol; d) ter sido publicado entre início de 2008 e fim de 2018 ; f) realizados na América Latina e Caribe.

O processo de análise foi realizado mediante as seguintes etapas: a) leitura de títulos e resumos; b) inclusão e exclusão de artigos conforme os critérios estabelecidos; c) leitura dos trabalhos completos pré-selecionados; d) análise final de artigos selecionados de acordo 
com os objetivos e critérios. Em todo o processo, houve um método de conferência e definição de consenso para cada etapa das buscas e análises. A amostra final foi de 20 artigos. Todo o processo de busca, seleção e análise pode ser observado no fluxograma Prisma (Figura 1).

Figura 1. fluxograma Prisma: fases de identificação, seleção, elegibilidade e inclusão da revisão sistemática

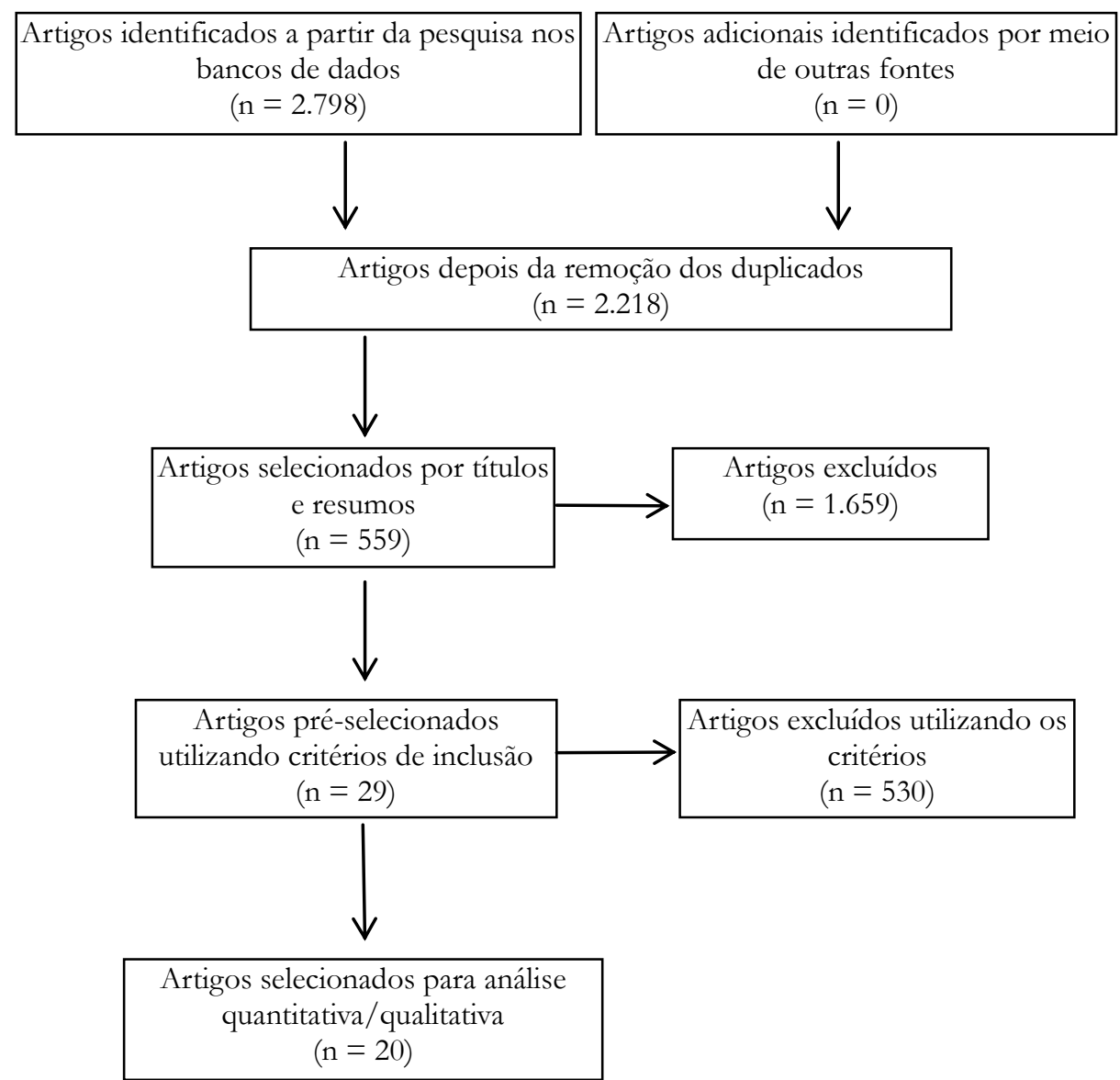

Fonte: Elaborada pelos autores.

\section{Resultado}

Foram encontrados artigos provenientes de oito países: Brasil $(n=11,55 \%)$, México $(n=3,15 \%)$, Argentina $(n=1,5 \%)$, Colômbia $(n=1,5 \%)$, Costa Rica $(n=1,5 \%)$, Cuba $(n=$ 1, 5\%), Panamá ( $n=1,5 \%)$, Peru $(n=1,5 \%)$. Destes, 13 artigos eram quantitativos (65\%); 
cinco, qualitativos (25\%); e dois com métodos mistos - qualitativos/quantitativos (10\%). Os anos com maior frequência de publicações foram 2011 e 2016 (Figura 2). Sobre os idiomas, 12 (60\%) estavam em português; oito (40\%), em espanhol; e cinco (25\%), em inglês, compreendendo que quatro artigos foram encontrados em mais de um idioma.

Figura 2. Ano das publicações selecionadas para análise

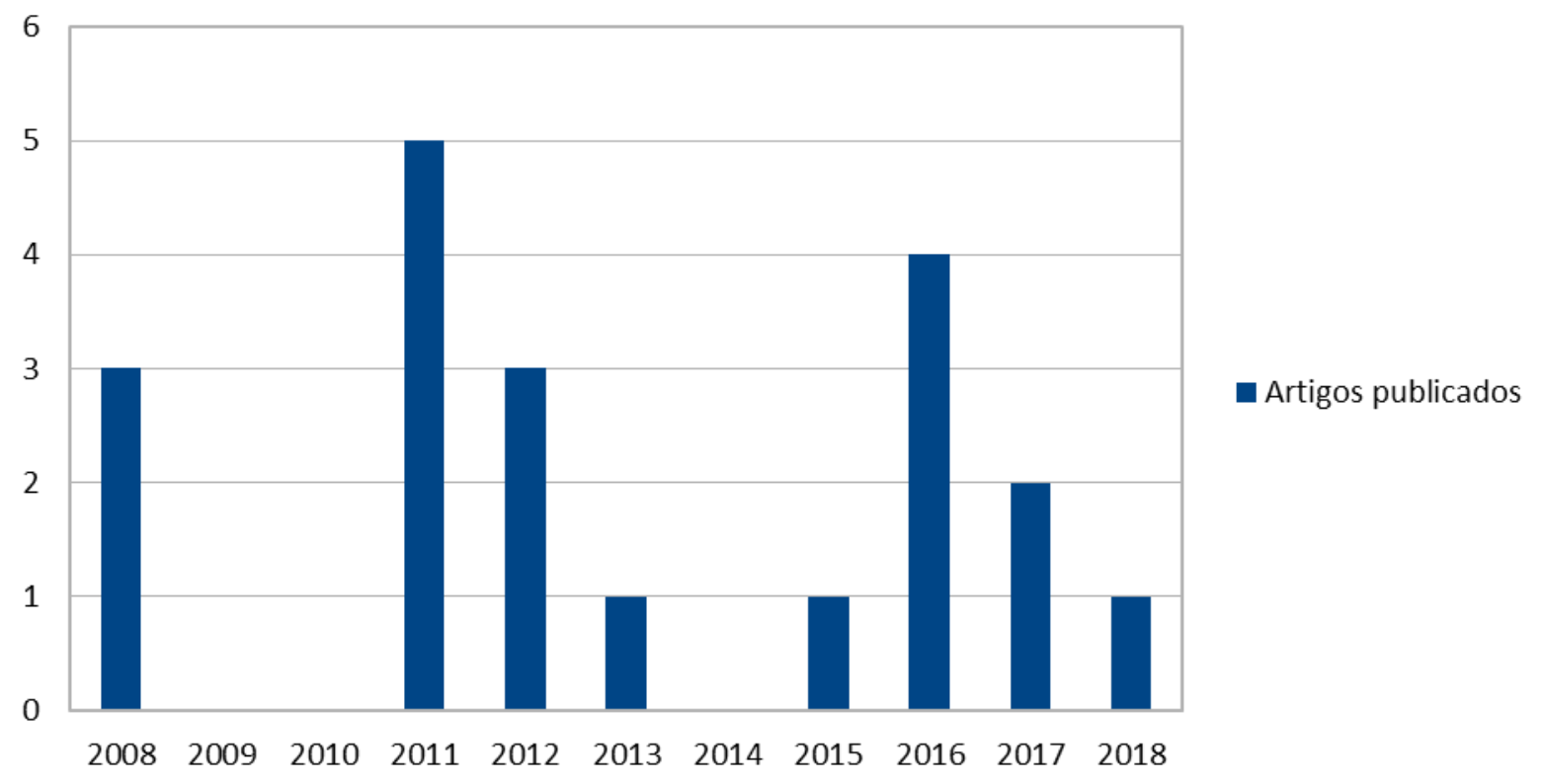

Fonte: Elaborada pelos autores.

No tocante aos bancos de dados, seis artigos foram encontrados em mais de uma plataforma: Lilacs ( $n=16,80 \%)$, PubMed ( $n=4,20 \%)$, SciELO $(n=4,20 \%)$, PePSIC $(n=1$, 5\%) e PsycINFO/PsycNET ( $n=1,5 \%)$. Observa-se uma grande variação de títulos de revistas, com maior concentração de estudos na Cadernos de Saúde Pública $(\mathrm{N}=3)$ e Revista LatinoAmericana de Enfermagem $(\mathrm{N}=2)$, conforme a Tabela 1.

Tabela 1. Títulos das Revistas em que os artigos foram publicados

\begin{tabular}{|l|l|}
\hline Revistas & N (\%) \\
\hline
\end{tabular}




\begin{tabular}{|l|l|} 
Cadernos de Saúde Pública & $3(15 \%)$ \\
\hline $\begin{array}{l}\text { Revista de Latino-Americana } \\
\text { Enfermagem }\end{array}$ & $2(10 \%)$ \\
\hline $\begin{array}{l}\text { Cadernos de Terapia Ocupacional da } \\
\text { UFSCar }\end{array}$ & $1(5 \%)$ \\
\hline Ciência \& Saúde Coletiva & $1(5 \%)$ \\
\hline Escola Anna Nery & $1(5 \%)$ \\
\hline Glob Public Health & $1(5 \%)$ \\
\hline Jornal Brasileiro de Pneumologia & $1(5 \%)$ \\
\hline Medwave & $1(5 \%)$ \\
\hline Pesquisas e Práticas Psicossociais & $1(5 \%)$ \\
\hline Psico USF & $1(5 \%)$ \\
\hline $\begin{array}{l}\text { Revista Americana de Medicina } \\
\text { Respiratória }\end{array}$ & $1(5 \%)$ \\
\hline Revista Brasileira de Saúde Ocupacional & $1(5 \%)$ \\
\hline Revista Ciencias de la Salud & $1(5 \%)$ \\
\hline Revista Colombiana de Psiquiatría & $1(5 \%)$ \\
\hline Revista de Saúde Pública & $1(5 \%)$ \\
\hline Salud Pública de México & $1(5 \%)$ \\
\hline $\begin{array}{l}\text { Revista Eletrônica Saúde Mental Álcool e } \\
\text { Drogas }\end{array}$ & $1(5 \%)$ \\
\hline Font: Elaborada pelos autores. & \\
\hline \multicolumn{1}{|c|}{ de } \\
\hline
\end{tabular}

Fonte: Elaborada pelos autores.

As substâncias mais estudadas foram álcool ( $n=12,60 \%)$, tabaco ( $n=9,45 \%)$, drogas ilícitas não identificadas $(n=2,10 \%)$ e drogas lícitas e ilícitas não identificadas $(\mathrm{n}=$ 1, 5\%). Como pode se observar na Tabela 2, foram realizadas categorizações sobre a população-alvo estudada: população/moradores de determinada região/zona rural, agricultores, jovens e adolescentes, moradores de território de reforma agrária, mulheres, pacientes homens e moradores em vilas rurais em diferentes altitudes.

Tabela 2. Classificação das populações-alvo

\section{Populações-alvo}


População/moradores de determinada região/zona rural

- Autores-chave e agentes da comunidade de Antioquia, Colômbia

- População ribeirinha do interior da Amazônia

- Residentes de uma área de migração agrícola em uma comunidade rural da Baixa Califórnia, México

- População rural de Lamarão, Brasil

- População urbana/rural acima de 15 anos de uma área rural da província de 1 (5\%)

Buenos Aires, Argentina

- Fumantes do Panamá

- Moradores de Parazinho, Brasil

\section{Agricultores}

- Fumicultores de São Lourenço do Sul-RS, Brasil

- Citricultores de Lagarto-SE, Brasil

- Cortadores de cana do oeste de São Paulo, Brasil

Jovens e adolescentes

$4(20 \%)$

- Jovens costarriquenhos, institucionalizados em duas Organizações não

Governamentais

$1(5 \%)$

$1(5 \%)$

$1(5 \%)$

- Pais e adolescentes escolares da zona rural de Barão do Triunfo-RS, Brasil

- Adolescentes da área rural de Novo Leon, México

- Adolescentes de zona rural versus zona urbana em Monterrei, México

Moradores de território de reforma agrária

$2(10 \%)$

- Moradores de nove assentamentos no estado do Rio Grande do Norte e seis do 2 (10\%) estado do Piuaí, Brasil

\section{Mulheres}

- Mulheres da zona rural de Teresina-PI, Brasil

Pacientes homens

- Homens hospitalizados na área rural de Cumanayagua, Cuba

Moradores em vilas rurais em diferentes altitudes

- Residentes de áreas rurais de diferentes altitudes do Peru

Fonte: Elaborada pelos autores.

Em relação aos objetivos e resultados encontrados nas pesquisas, utilizamos as categorias de população-alvo e realizamos uma análise qualitativa com o propósito de traçar um cenário geral sobre os estudos, a fim de chamar atenção para alguns dados ou características destes. Na primeira categoria, "População/moradores de determinada região/zona rural", três estudos tiveram objetivos de observar ou determinar a prevalência 
de tabagismo nas regiões dos trabalhos, um com foco nos altos ricos cardiovasculares da população; outro em analisar as relações socioeconômicas e sociodemográficas e a compra de produtos de tabaco ilegais e; o próximo, estudar as características locais para subsidiar a elaboração de um programa de controle do tabagismo. Os resultados indicaram associação do tabagismo com falta de exercício físico e consumo de álcool, além de uma alta proporção de sujeitos com alto risco de doenças cardiovasculares continuarem fumando (Landea et al., 2011 ). A venda de cigarros ilegais ocorreu em maior número na amostra urbana, em relação à rural, mas está principalmente ligada a fatores socioeconômicos (Herrera-Ballesteros, Zúñiga, Moreno, Gómez \& Roa-Rodríguez, 2017). Por último, houve prevalência do sexo masculino em relação ao tabagismo, grande parte começa a fumar antes dos 12 anos de idade, a condição econômica desfavorável aparece como fator agravante, além de existir forte associação com doenças respiratórias e com ingestão de álcool (Falcão \& Costa, 2008).

Ainda nesta primeira categoria, dois artigos realizaram estudos específicos sobre o consumo de álcool. O primeiro com o objetivo de adaptar e validar o Audit (Alcohol Use Disorders Identificantion Test) para uma população ribeirinha no Amazonas; e o outro objetivou compreender o significado que os habitantes de uma comunidade rural tinham em relação ao consumo de álcool. Os resultados mostram que o Audit adaptado pode ter confiabilidade interna adequada (Moretti-Pires \& Corradi-Webster, 2011); e que o consumo de álcool é um evento emocional, o qual permite às pessoas a expressão de sentimentos e emoções, especialmente quando a cultura não permite esse tipo de manifestação. Esse consumo se apresenta como alternativa de afrontamento a situações de dor (Páez-Zapata \& Posada, 2015).

Por fim, os últimos dois textos desta primeira categoria tiveram diferentes objetivos, um de avaliar uma atividade de ensino e extensão em uma comunidade rural por meio de um diagnóstico participativo. Os resultados apresentam uso de agrotóxicos e de pulverização aérea, além do uso de drogas lícitas e ilícitas como fatores ameaçadores à vida, que estariam também associados à cultura alimentar, ao uso incorreto de equipamentos de proteção e à falta de lazer e transporte. Por outro lado, fatores promotores de vida na comunidade foram identificados, tais como: diversidade na produção de alimentos, 
liberdade e segurança, união e organização da comunidade, geração de empregos e natureza (Carneiro et al., 2012). O segundo estudo examina a familiaridade com o cenário de drogas e a violência, e seus resultados mostram que há uma relação positiva entre violência e o cenário de drogas com uma associação a movimentos migratórios campocidade e cidade-campo (Volkmann et al., 2013).

Na segunda categoria, "Agricultores", os fumicultores de uma mesma região são observados em dois estudos, tanto com sua relação com o álcool quanto com o tabaco. Os resultados mostram que o consumo de risco e o beber pesado têm prevalência em ambas as regiões, e que nesse contexto, o nível de diversificação da renda, as intempéries causadas pelo clima, o endividamento, as longas jornadas de trabalho pesado e os riscos ligados à manipulação de agrotóxicos devem ser considerados na cadeia causal (Fávero, Meucci, Faria, Fiori \& Fassa, 2018). Já o consumo de tabaco está fortemente associado aos homens e às variáveis socioeconômicas como idade, escolaridade, renda, ser bebedor pesado, tempo de trabalho na fumicultura e tempo de exposição a pesticidas. O trabalho em si é um fator de risco, sendo que a naturalização deste e a herança cultural podem ser fatores comuns e exigem uma abordagem conjunta (Fiori, Faria, Meucci \& Fassa, 2016).

Observou-se a qualidade de vida dos cortadores de cana, sendo que os tabagistas têm maiores índices de desistência no trabalho ao fim do período de entressafra. $\mathrm{O}$ aspecto físico, social e emocional de não tabagistas é positivado em relação aos tabagistas no período de safra (Carvalho Junior et al., 2012).

Por último, procurou-se entender a saúde dos citricultores a partir da estrutura agrária, refletindo na saúde mental do trabalhador e expresso por meio do abuso de álcool. Os resultados mostraram que é comum encontrar trabalhadores no posto de trabalho sob efeito de álcool, e a maioria alega que bebe em exames clínicos; além disso, a maioria apresentou alteração no fígado, no entanto, não se sabe se o que causou essa alteração foi o uso de álcool ou do agrotóxico (Santos \& Menta, 2016).

$\mathrm{Na}$ terceira categoria, "Jovens e Adolescentes", dois estudos foram realizados na mesma região do México, no mesmo ano e com objetivos semelhantes: compreender o consumo de álcool e tabaco e o efeito na autoestima dos jovens. Os resultados do primeiro 
destacaram que os homens consomem mais que as mulheres; o álcool é mais consumido que o tabaco; e o consumo apresenta uma correlação negativa com a autoestima (Garcia, Aguilar \& Facundo, 2008). O outro estudo aponta diferença significativa no consumo de tabaco entre estudantes do ensino fundamental de áreas urbanas, em comparação aos estudantes da área rural. Houve também uma correlação negativa entre padrão de consumo de álcool e tabaco e autoestima (Maldonado, Pedrão, Castillo, García \& Rodríguez, 2008). Ainda entre essa população, houve um estudo que correlacionou o padrão de consumo de drogas dos pais e filhos adolescentes. Os resultados apontaram para uma correlação positiva entre os dois grupos, havendo uma discussão sobre as expectativas culturais locais (Raphaelli, Azevedo \& Hallal, 2011). Por último, uma pesquisa investigou a percepção de jovens institucionalizados, provenientes de zonas rurais e afastados dos pais, sobre o consumo de drogas ilícitas. Obteve-se que a autopercepção dos jovens está focada nos processos de mudanças e experimentação que os fazem tomar decisões, como consumir drogas. As relações conflituosas com os pais são fatores agravantes nessa autopercepção, já os amigos da instituição são vistos como parte de uma rede de apoio (Murillo-Castro \& Miasso, 2011 ).

$\mathrm{Na}$ categoria "Moradores de território reforma agrária", os dois estudos foram realizados na mesma região e mesmo grupo de pesquisadores, com objetivos semelhantes de investigar o consumo de álcool com as condições de vida local; um com foco nas comorbidades psiquiátricas e o outro na formação dos profissionais que atuam na área. Os resultados mostram que a pobreza é um fator decisivo na prevalência de transtornos mentais e abuso de drogas, além de agudizar as iniquidades sociais, tornando os assentados rurais uma população de risco. Contudo, as inter-relações entre desigualdades sociais e o processo saúde-doença-cuidado são complexas e perpassam pelas histórias de vida, dinâmicas familiares, formas de organização social, características geográficas, que vão construindo as singularidades dos processos de vida e vivências do sofrimento psíquico (Dimenstein, Macedo, Leite, Dantas \& Silva, 2017). No outro estudo, identificou-se ainda que homens os consomem quatro a cinco vezes mais que as mulheres, de forma problemática; conhecer a realidade do uso de álcool mostrou que as condições de vida e as fragilidades na 
infraestrutura, gênero, escolaridade, renda, ocupação, acesso aos serviços de saúde e educação são fatores influenciadores do consumo problemático nesses contextos, indicando a necessidade de reorientar práticas e a formação profissional (Macedo, Dimenstein, Leite \& Dantas, 2016).

Na categoria "Mulheres", o objetivo do estudo único foi descrever e analisar os relatos de mulheres em uso prejudicial de bebidas alcoólicas. Os resultados mostraram uma trajetória de vida sofrida, desde a infância permeada por alcoolismo; as amizades, residência próxima a bares e más condições de trabalho são fatores de risco, além disso, os relatos mostram que há prioridade em relação à compra de bebidas e que existe reconhecimento por parte das mulheres em relação aos prejuízos orgânicos e desempenho profissional devido ao consumo (Monteiro, Dourado, Graça Júnior \& Freire, 2011).

Já na categoria "Pacientes homens", também com estudo único, o objetivo foi determinar os fatores de risco que com maior frequência se associam ao alcoolismo. São eles: ser maior de 35 anos de idade, não aceitar a própria doença, terem pais alcoolistas ou família disfuncional e estarem envolvidos em problemas judiciais (Pereira, López, Juviel, Suárez \& Fernández, 2013).

Por último, a categoria "Moradores em vilas rurais em diferentes altitudes" traz um estudo que busca determinar a relação entre altitude e dependência alcoólica. Os resultados mostram associação positiva entre extrema pobreza e alcoolismo, além de identificar moradores de baixas altitudes como população com maior frequência de dependentes de álcool. O fato de ser mulher e morar em altas altitudes foi associado negativamente com a frequência de consumo de álcool (Quiñones-Laveriano, Espinoza-Chiong, Scarsi-Mejia, Rojas-Camayo \& Mejia, 2016).

\section{Discussão}

Sobre a busca, o Lilacs se mostrou como um grande referencial de base de dados para o tema, enquanto o PsycINFO/PsycNET foi irrelevante na busca, visto que o único artigo encontrado nessa base de dados também foi visto no PubMed. Foram encontradas 17 
diferentes revistas para 20 artigos, apenas Cadernos de Saúde Pública $(\mathrm{n}=3,15 \%)$ e Revista Latino-Americana de Enfermagem $(\mathrm{n}=2,10 \%)$ tiveram mais de um estudo, o que mostra que o tema está diluído sem um padrão claro e que não há revistas que podemos adotar como referência para estudos na área, o que pode dificultar o avanço e compreensão dos problemas que cercam o assunto.

Devemos inicialmente destacar que a amostra final contou com 11 artigos (55\%) do Brasil e que seis países (30\%) têm estudos únicos, além de faltar representatividade de países que não apareceram na busca, dificultando análises mais homogêneas. No entanto, ao estudar os meios rurais, entendemos que a heterogeneidade do campo é uma característica que perpassa a formação cultural de seus contextos e passa por complexas redes de sociabilidades entre os indivíduos em suas relações com o material e o simbólico (Silva \& Macedo, 2017); podemos então nos apropriar do conteúdo produzido aqui para compreender nossa realidade ou a de onde estivermos colocados e/ou nos colocado como profissionais, tendo assim material para adaptar os métodos e teorias ao contexto produzido por seus autores.

Ainda sobre ruralidade, podemos perceber que não existe consenso do que é rural, alguns compreendem que o espaço urbano de determinados municípios compõe uma área rural, uma vez que esses municípios podem carregar heranças culturais, além de ter base em atividades agrícolas e/ou pecuária (Fiori et al., 2016, Landea et al., 2011, Raphaelli et al., 2011, \& Falcão \& Costa, 2008). Outro estudo faz distinção de diferentes maneiras: diferenciação entre urbano, rural e indígena, marcando a área indígena como categoria independente (Herrera-Ballesteros et al., 2017).

Adota-se, também, a definição de comunidades rurais não centrada na questão geográfica, compreendendo o trabalho como categoria importante para determinar o fazer em contextos rurais, algumas vezes marcado por períodos migratórios, como os períodos de safra e entressafra, que identificam esses trabalhadores como população rural (Carvalho Junior et al., 2012). Em contrapartida, também é visto que o trabalhador em contexto rural só poderia ser considerado na categoria de população rural se morasse no campo (Fávero et al., 2018). 
A categoria geográfica aparece claramente a partir da altitude (Quiñones-Laveriano et al., 2016), mas também pode ser percebida a partir das regionalidades. Alguns estudos não identificam como definiram o que é rural, mas aparentemente seguem o raciocínio de adotar características geográficas, populacionais e/ou culturais. Tudo isso reforça a heterogeneidade nesses contextos e a necessidade de estudos nessas regiões a fim de identificar possíveis variáveis e relações que, às vezes, são encobertas por preconceitos.

Outro ponto é a maior quantidade de estudos quantitativos ( $n=13,65 \%$ ), além de outros dois estudos (10\%) quali/quanti. A "questão das drogas" a partir de tais estudos, em geral, apresentou características epidemiológicas que buscaram identificar ou determinar a prevalência de padrões de consumo. Todavia, alguns buscam sair dessa lógica, compreendendo os processos inerentes ao consumo do álcool a partir de uma visão cultural (Pereira, 2013) e discutindo que medir e qualificar o consumo é complexo, devido aos aspectos culturais, sendo complexa a classificação padronizada e universal de dano por uso de substância (Fávero et al., 2018). Seguindo esse pensamento, podemos perceber que a heterogeneidade não parte apenas dos contextos rurais, mas também do contexto do consumo de substâncias e das características que as cercam.

A maior quantidade de estudos sobre álcool e tabaco era esperada, desde a escolha dos termos. No entanto, a escassez de estudos sobre outras drogas é importante ser destacada, apontando para uma lacuna na literatura. A escolha de três estudos (15\%) de não identificar as drogas, apenas categorizar como "drogas ilícitas não identificadas" ou "drogas lícitas e ilícitas não identificadas", oferece pouco para a compreensão do uso de outras substâncias (Volkmann et al., 2013; Carneiro et al., 2012; \&Murillo-Castro \& Miasso, 2011).

Sobre o tabaco, podemos perceber maior diferenciação entre a substância quanto à legalidade do produto, o tabaco ilegal aparece diretamente ligado a doenças, e as populações mais pobres tendem a consumir em maior escala esse tipo de produto (HerreraBallesteros et al., 2017). A pobreza, característica da América Latina condescende com esse consumo, e o cenário que envolve o tabaco ilegal, ameaça ainda mais a saúde pública, para além da legislação e fiscalização se apresenta necessário mecanismos psicossociais. 
Nos resultados, a população do sexo masculino acompanha a tendência de outros estudos na área, ao revelar que os homens bebem mais que as mulheres (UNODC, 2016). No entanto, podemos destacar o trabalho duro e com intempéries como agravante, uma vez que os agricultores trazem uma herança cultural de exposição e tempo de trabalho que muitas vezes está vinculada ao uso de agrotóxicos (Riquinho \& Hennington, 2016). Em algumas regiões, essa exposição pode ter diferentes agravantes, como no Sul do Brasil, onde fumicultores vivem um cotidiano de (re)normatizações em confronto com as normas e valores instituídos pela indústria do tabaco, que cria mecanismos de controle e exploração, havendo casos de isolamento social e endividamento devido ao trabalho. A dificuldade desses trabalhadores rurais para defender seus direitos é tocante, uma vez que se cria um ciclo de exploração, dependência e subordinação (Riquinho \& Hennington, 2016).

Outro grupo de destaque nos resultados, jovens e adolescentes, também segue os apontamentos da literatura (UNODC, 2016), aqui as questões que envolvem a autoestima, o desenvolvimento emocional e as relações parentais são centrais nos estudos que aparecem. Além disso, em comparação ao adolescente urbano, o adolescente rural está mais propenso a ajudar na economia familiar, como aponta Maldonado et al. (2008), dando destaque aos fatores socioeconômicos que cercam essas relações.

Por último, ressaltamos que a pobreza surge como forte condição para o abuso de álcool e tabaco nesses contextos, tornando-se um grande desafio para os profissionais. $\mathrm{O}$ conhecimento das necessidades dos moradores rurais é extremamente desafiante, como asseveram Macedo et al. (2016), que apostam em um modelo de formação profissional em que as determinações das necessidades sociais e de saúde mental das populações rurais perpassam por dispositivos de escuta e intervenção sobre os processos psicológicos e psicossociais mobilizados pelas condições de vida e situações de pobreza.

\section{Considerações finais}

Nesta análise, percebemos como se encontra o cenário do consumo de drogas em contextos rurais na América Latina e Caribe na literatura científica, tanto as drogas quanto a 
ruralidade se apresentam como categorias heterogêneas que, ao serem cruzadas, se apresentam como um desafio aos profissionais. Os resultados também seguem essa tendência heterogênea, por isso a importância da discussão do tema na saúde pública e no meio acadêmico para fortalecer instituições públicas e embasar o fazer dos profissionais da área.

As condições socioeconômicas da América Latina nesse cenário são perpassadas pela pobreza, que se torna variável fundamental para a investigação. Um olhar atento ao contexto deve envolver a história, a cultura, a geografia, o território e a população, norteando onde o consumo de determinada substância entra na investigação e quais aspectos existem nessas relações.

Esse olhar ampliado fundamenta pesquisas mais sólidas e permite ao pesquisador se livrar de preconceitos teóricos ou do senso comum e desenvolver metodologias de pesquisas que levem em consideração essas variáveis. Estimular o desenvolvimento de pesquisas acerca desse tema é fundamental, compreendendo que o que acontece nas cidades perpassa pelo campo, e que não existe entendimento dos processos psicossociais quando os contextos rurais estão desprendidos da análise.

\section{Referências}

Carneiro, F. F., Hoefel, M. G., Silva, M. A. M., Nepomuceno, A. R., Vilela, C., Amaral, F. R., Carvalho, G. P. M., Batista, J. L., \& Lopes, P. A. (2012). Mapeamento de vulnerabilidades socioambientais e de contextos de promoção da saúde ambiental na comunidade rural do Lamarão, Distrito Federal, 2011. Revista Brasileira de Saúde Ocupacional, 37(125), 143-148.

Carvalho Junior, L. C. S., Ramos, E. M. C., Toledo, A. C., Ceccato, A. D. F., Macchione, M., Braga, A. L. F., \& Ramos, D. (2012). Avaliação da qualidade de vida relacionada à saúde de cortadores de cana-de-açúcar nos períodos de entressafra e safra. Revista de Saúde Pública, 46(6), 1058-1065.

Dimenstein, M. D. B., Macedo, J. P. S., Leite, J. F., Dantas, C. M. B., \& Silva, M. P. R. (2017). Iniquidades sociais e saúde mental no meio rural. Psico USF, 22(3), 541-553.

Dimenstein, M. D. B., Dalla Vecchia, M., Macedo, J. P., \& Bastos, I. B. (2018). (Des)Occupation 
of Urban and Rural Spaces, Gentrification and Drug Use. In Drugs and Social Context (pp. 89-110). Switzerland: Springer.

Falcão, T. J. O., \& Costa, I. C. C. (2008). O tabagismo em um município de pequeno porte: um estudo etnográfico como base para geração de um programa de saúde pública. Jornal Brasileiro de Pneumologia, 34(2), 91-97.

Fávero, J. L., Meucci, R. D., Faria, N. M. X., Fiori, N. S., \& Fassa, A. G. (2018). Consumo de bebida alcoólica entre fumicultores: prevalência e fatores associados. Ciência \& Saúde Coletiva, 23(3), 871-882.

Fiocruz. (2017). III Levantamento Nacional sobre o Uso de Drogas pela População Brasileira. Ministério da Saúde.

Fiori, N. S., Faria, N. M. X., Meucci, R. D., \& Fassa, A. G. (2016). Prevalência e fatores associados ao tabagismo em fumicultores do Sul do Brasil. Cadernos de Saúde Pública, 32(7).

Galvão, L. A. C., Finkelman, J., \& Henao, S. (2011). Determinantes ambientais e sociais da saúde. Washington: OPAS.

Galvão, T. F., Pansani, T. S. A., \& Harrad, D. (2015). Principais itens para relatar revisões sistemáticas e meta-análises: a recomendação Prisma. Epidemiologia e Serviços de Saúde, 24(2), 335-342.

Garcia, N. A. A., Aguilar, L. R., \& Facundo, F. R. G. (2008). Efecto de la autoestima sobre el consumo de tabaco y alcohol em adolescentes del área rural de Nuevo León, México. SMAD. Revista eletrônica saúde mental álcool e drogas, 4(1), 00.

Herrera-Ballesteros, V. H., Zúñiga, J., Moreno, I., Gómez, B., \& Roa-Rodríguez, R. (2017). Factores socioeconómicos asociados con la compra ilegal de productos de tabaco en Panamá. Salud Pública de México, 59(1), 88-96.

IBGE. (2017). Classificação e caracterização dos espaços rurais e urbanos no Brasil: uma primeira aproximação. Rio de Janeiro: IBGE.

Landea, M. R., Salazar, M. R., Marillet, A. G., Novello, M. A., Carbajal, H. A., \& Echeverría, R. F. (2011). Prevalencia de tabaquismo y factores de riesgo cardiovascular en casco urbano de una localidad rural de la Provincia de Buenos Aires. Revista Americana de Medicina Respiratoria, 1 1(3), 110-116.

Liberati, A., Altman, D. G., Tetzlaff, J., Mulrow, C., Gotzsche, P. C., Ioannidis, J. P. A., Clarke, M., Devereaux, P. J., Kleijnen, J., \& Moher, D. (2009). The PRISMA Statement for Reporting Systematic Reviews and Meta-Analyses of Studies That Evaluate Health Care Interventions: Explanation and Elaboration. PloS Med, 6(7). 
Macedo, J. P., Dimenstein, M., Leite, J., \& Dantas, C. (2016). Condições de vida, pobreza e consumo de álcool em assentamentos rurais: desafios para atuação e formação profissional. Pesquisas e Práticas Psicossociais, $17(3), 552-569$.

Maldonado, R. M., Pedrão, L. J., Castillo, M. M. A., García, K. S. L., \& Rodríguez, N. N. O. (2008). Auto-estima, auto-eficácia percebida, consumo de tabaco e álcool entre estudantes do ensino fundamental, das áreas urbana e rural, de Monterrey, Nuevo León, México. Revista latino-americana de enfermagem, 16(n.esp), 614-620.

Mendes, K. T., Ronzani, T. M., \& Paiva, F. S. (2019). Poverty, Stigma, and Drug Use: Reflections about a Perverse Relation. In Psychosocial Implications of Poverty (pp. 7790). Switzerland: Springer.

Monteiro, C. F. S., Dourado, G. O. L., Graça Júnior, C. A. G., \& Freire, A. K. N. (2011). Relatos de mulheres em uso prejudicial de bebidas alcoólicas. Escola Anna Nery, 15(3), 567572.

Moretti-Pires, R. O., \& Corradi-Webster, C. M. (2011). Adaptação e validação do Alcohol Use Disorder Identification Test (Audit) para população ribeirinha do interior da Amazônia, Brasil. Cadernos de Saúde Pública, 27(3), 497-509.

Murillo-Castro, L., \& Miasso, A. I. (2011). Visión de jóvenes Costarricenses, de zonas rurales, en un programa de rehabilitación, sobre el consumo de drogas. Revista LatinoAmericana de Enfermagem, 796-803.

Páez-Zapata, E., \& Posada, I. C. (2015). Significados al consumo de alcohol en habitantes de una comunidad rural, Antioquia, Colombia, 2010-2011. Revista Ciencias de la Salud, 13(1), 77-90.

Pereira, F. I. H., López, G. M., Juviel, A. I. F., Suárez, V. G., \& Fernández, Y. H. (2013). Alcoholismo y factores de riesgo: estudio descriptivo de corte transversal en área rural de Cumanayagua, Cuba. Medwave, 13(1).

Quiñones-Laveriano, D. M., Espinoza-Chiong, C., Scarsi-Mejia, O., Rojas-Camayo, J., \& Mejia, C. R. (2016). Altitud geográfica de residencia y dependencia alcohólica en pobladores peruanos. Revista colombiana de psiquiatría, 45(3), 178-185.

Raphaelli, C. O., Azevedo, M. R., \& Hallal, P. C. (2011). Associação entre comportamentos de risco à saúde de pais e adolescentes em escolares de zona rural de um município do Sul do Brasil. Cadernos de saúde pública, 27(12), 2429-2440.

Riquinho, D. L., \& Hennington, E. A. (2016). Sistema Integrado de produção de tabaco: saúde, trabalho e condições de vida de trabalhadores rurais no Sul do Brasil. Cadernos de saúde pública, 32(12). 
Ronzani, T. M. (2018). The Context of Drug in the Consumer Society. In Drugs and Social Context (pp. 3-14). Switzerland: Springer.

Ronzani, T. M., Mendes, K., de Oliveira, C., \& Leite, J. (2019). Contextos Rurais e Psicologia Comunitária: um encontro possível e necessário. In M. N. de Carvalho-Freitas, L. C. Freitas \& T. C. Pollo (Orgs.). Instituições, saúde e sociedade: contribuições da Psicologia (pp. 59-79). Belo Horizonte: EdUEMG. Recuperado de http://eduemg.uemg.br/images/livros-pdf/catalogo-

2019/Instituicoes_saude_e_sociedade_eletronico.pdf.

Santos, A. C., \& Menta, S. A. (2016). Refletindo a interface entre trabalho rural e saúde mental dos trabalhadores da citricultura. Cadernos de Terapia Ocupacional da UFSCar, 24(4), 765-775.

Savassi, L. C. M., Almeida, M. M., Floss, M., \& Lima, M. C. (2018). Saúde no caminho da roça. Rio de Janeiro: Fiocruz.

Silva, K. B., \& Macedo, J. P. (2017). Inserção e trabalho de psicólogas/os em contextos rurais: interpelações à Psicologia. Revista de Psicologia, 8(2), 146-154.

UNODC. (2017). Prevention of Drug Use and Treatment of Drug Use Disorders in Rural Settings. New York: United Nations. Retrieved from https:/ / www.unodc.org/documents / 17-01904_Rural_treatment_ebook.pdf.

UNODC. (2016). World Drug Report. New York: United Nations. Retrieved from https://www.unodc.org/doc/wdr2016/WORLD_DRUG_REPORT_2016_web.pdf.

Volkmann, T., Fraga, M. A., Brodine, S. K., Iñiguez-Stevens, E., Cepeda, A., Elder, J. P., Garfein, R. S., \& VIIDAI Team. (2012). Drug-Scene Familiarity and Exposure to Gang Violence among Residents in a Rural Farming Community in Baja California, Mexico. Glob Public Health, 8(1), 65-78. 